\title{
Temporomandibular disorders among medical students in China: prevalence, biological and psychological risk factors
}

Jing $\mathrm{Wu}^{1 \dagger}$, Zelun Huang ${ }^{2+}$, Yong Chen ${ }^{3}$, Yifan Chen ${ }^{1}$, Zhongqiang Pan ${ }^{4}$ and Yu Gu${ }^{5^{*}}$

\begin{abstract}
Background: The purpose of the present study is to evaluate the prevalence of temporomandibular disorders (TMD) and their associated biological and psychological factors in Chinese university students.

Methods: A total of 754 students were included from Zunyi Medical University, each participant completed questionnaires and clinical examinations according to the Diagnostic Criteria for Temporomandibular Disorders.

Results: The overall prevalence of TMD was $31.7 \%$ among medical students. Subjects with TMD had a high prevalence of bruxism, empty chewing, unilateral chewing, chewing gum, anterior teeth overbite, anterior teeth overjet, depression, anxiety, and sleep disturbance. Moreover, sleep bruxism, empty chewing, unilateral chewing, anterior teeth overbite, depression, and anxiety were the strongest risk factors for TMD.

Conclusions: Individuals with TMD have a high prevalence of psychological distress and oral parafunctional habits. Except for the psychological factors associated with TMD, bruxism, abnormal chewing, and malocclusion also shared similar risks for TMD.
\end{abstract}

Keywords: Temporomandibular disorders, Psychological factors, Parafunctional habits

\section{Introduction}

Temporomandibular disorders (TMD) are common clinical musculoskeletal pain syndromes involving the surrounding musculature and temporomandibular joint, mainly characterized by limited joint movement, articular sounds, or joint and muscular pain [1]. The prevalence of TMD is quite variable from 7 to $30 \%$, and symptoms of TMD are common among adults aged $20-40$ years old $[2,3]$. When TMD manifests as severe clicking, pain, or limited mobility of joints, it consistently affects the quality of life and forces patients to seek help. Due to the high prevalence and high cure rates of TMD, it is important

\footnotetext{
*Correspondence: gylookfish@hotmail.com

${ }^{\dagger}$ Jing Wuand Zelun Huang are Co-first authors of this study

${ }^{5}$ Department of Stomatology, Zhuhai Campus of Zunyi Medical

University, 368 Jinwan Road, Zhuhai 519041, Guangdong Province, China

Full list of author information is available at the end of the article
}

for TMD to identify TMD patients and pay attention to complex etiologies.

The etiology and pathophysiology of TMD have been considered to be multifactorial and complex, consisting of trauma, stress, parafunctional habits, as well as psychological, hereditary, and occlusal factors [3, 4]. The most acceptable theory of TMD etiology is the biopsychological-based model and the combination of initiating and persistent factors interference with enhancing TMD progression [5-7]. Parafunctional habits, such as bruxism and clenching, are biological etiology factors for symptoms observed commonly in TMD [8-10]. In most cases, parafunctional habits mediating the increased tension of the masticatory musculature are the reason for TMD symptoms, and persistent parafunction contributes to worsening TMD symptoms. And associations between malocclusions and clinical signs of TMD were

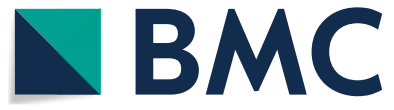

(c) The Author(s) 2021. Open Access This article is licensed under a Creative Commons Attribution 4.0 International License, which permits use, sharing, adaptation, distribution and reproduction in any medium or format, as long as you give appropriate credit to the original author(s) and the source, provide a link to the Creative Commons licence, and indicate if changes were made. The images or other third party material in this article are included in the article's Creative Commons licence, unless indicated otherwise in a credit line to the material. If material is not included in the article's Creative Commons licence and your intended use is not permitted by statutory regulation or exceeds the permitted use, you will need to obtain permission directly from the copyright holder. To view a copy of this licence, visit http://creativecommons.org/licenses/by/4.0/. The Creative Commons Public Domain Dedication waiver (http://creativeco mmons.org/publicdomain/zero/1.0/) applies to the data made available in this article, unless otherwise stated in a credit line to the data. 
also detected in several studies, particularly, TMDs have associations with functional occlusion factors [11, 12]. Furthermore, TMD patients have heightened levels of depression, anxiety, and stress, as well as somatic awareness dysfunction, and diverse psychological factors have been considered as potential risk factors for the development of TMD [13-15]. In addition, sleep disturbance, including insomnia, sleep disruption, or insufficiency, is found to be a possibly associated factor for TMD $[16,17]$. Comorbidities of psychologic distress and sleep disturbance are not uncommon in TMD.

Based on the diverse and complex etiology of TMD, the identification of TMD symptoms as well as associated factors would contribute to promoting abilities to detect the earlier stage of TMD, and early interventions ameliorate the TMD pain and improve the quality of life. Therefore, this study was aimed to determine the prevalence of TMD and its association with biological and psychological factors in medical university students.

\section{Methods}

\section{Participants}

This research was conducted in the Zhuhai campus of Zunyi Medical University, students were selected by simple random sampling. The inclusion criteria were as follows: (1) The dentition belongs to the permanent dentition, and the second molar is fully erupted; (2) No history of orthodontic treatment; (3) Volunteer to participate in research, and cooperate with completing a series of questionnaires and clinical examination. And the exclusion criteria were as follows: (1) Diagnosis of other orofacial pain disorders, polyarthritis, and other rheumatoid disease; (2) Diagnosis of neurologic and neuropsychiatric diseases. Each participant was required to complete a series of questionnaires, including oral behaviors checklist, TMD symptom questionnaire, psychological questionnaire, and sleep status questionnaire. In addition, each participant completed a clinical examination according to the guidelines of the Diagnostic Criteria for TMD (DC/TMD) to identify participants having TMD [18]. A total of 754 participates were included in the current study and written informed consent was obtained from all subjects. This study was approved by the ethical committee of the Zunyi Medical University (2019-H007).

\section{Assessment of TMD}

Oral behaviors were gathered in detail through the questionnaire, containing sleep bruxism, awake bruxism, empty chewing, unilateral chewing, and chewing gum [18]. The TMD symptom questionnaire and clinical examination were conducted based on the guidelines of the DC/TMD [18]. The symptom questionnaire of the DC/TMD involves 14 items relating to characteristics of TMD symptoms (specifically facial pain, headaches, temporomandibular joint sounds, temporomandibular joint closed and open locking) and is designed to gather necessary information for deriving the DC/TMD Axis I diagnosis (Additional file 1). Participants are involved in a protocolised clinical evaluation by trained dental specialists according to the clinical examination form in DC/TMD. TMD diagnoses are subsequently made based on the symptom questionnaire, clinical examination form and the DC/TMD diagnostic algorithms. And according to the TMD diagnosis, patients were divided into three groups: the pain-related TMD, the intra-articular TMD, and the combined TMD.

\section{Assessment of psychological factors}

The psychological questionnaires comprised the Patient Health Questionnaire-9 (PHQ-9) and the Generalized Anxiety Disorder Scale-7 (GAD-7), while sleep disturbance was evaluated by the Pittsburgh Sleep Quality Index (PSQI). The PHQ-9 has been adopted widely as a screening and diagnostic tool for the depressive disorder [19]. Depression was classified into five grades according to the computed scores: normal (0-4 scores), mild (5-9 scores), moderate (10-14 scores), severe (15-19 scores), and extremely severe (20-27 scores). The GAD-7 has composite reliability as well as criterion-related validity for assessing generalized anxiety disorder [20]. Anxiety was also classified into five grades: normal (0-4 scores), mild (5-9 scores), moderate (10-13 scores), severe (1418 scores), and extremely severe (19-21 scores). The PSQI was a self-rating sleep quality scale developed by Buysse et al. [21], which consisted of seven indicators, including sleep quality, sleep latency, sleep duration, habitual sleep efficiency, sleep disturbance, use of sleep medicine, and daytime dysfunction. Each indicator was scored on a scale of $0-3$, and the cumulative score of each indicator was the total score of PSQI, which ranged from 0 to 21 . The higher scores represented the worse sleep quality.

\section{Statistical analysis}

All data were conducted using SPSS software version 22 . The prevalence data were presented as percentages. The chi-square test and the independent-samples t-test were used to compare the prevalence among participates with or without TMD. Correlations between TMD and biological and psychological characteristics were assessed using the spearman test. The logistic regression analysis was 
used to determine possible risk factors for TMD. $p<0.05$ was considered to be statistically significant.

\section{Results}

\section{Prevalence of TMD}

A total of 754 students, who completed the questionnaire and the clinical examination form, were included in this study. Of the 754 students, there were 354 males and 400 females, aged $19(18,19)$ years. The overall prevalence of TMD was $31.7 \%$, showing no significant gender difference. Orofacial pain and joint noise were the most common symptoms of TMD with the incidence of $57.3 \%$ and $50.6 \%$, while headache and locking had the low incidence of $13.8 \%$ and $33.9 \%$ (Fig. 1). Moreover, the participants were divided into three groups as the pain-related TMD, the intra-articular TMD, and the combined TMD, with the respective prevalence of $15.5 \%, 66.9 \%$, and $17.6 \%$.

\section{Biological and psychological characteristics of TMD}

As shown in Table 1, students with TMD had a high prevalence of sleep bruxism, awake bruxism, empty chewing, unilateral chewing, and chewing gum, compared to those without TMD. As for occlusions, a significantly higher prevalence of anterior teeth overbite and anterior teeth overjet was observed in students with TMD. In the psychological aspect, a significantly higher prevalence of

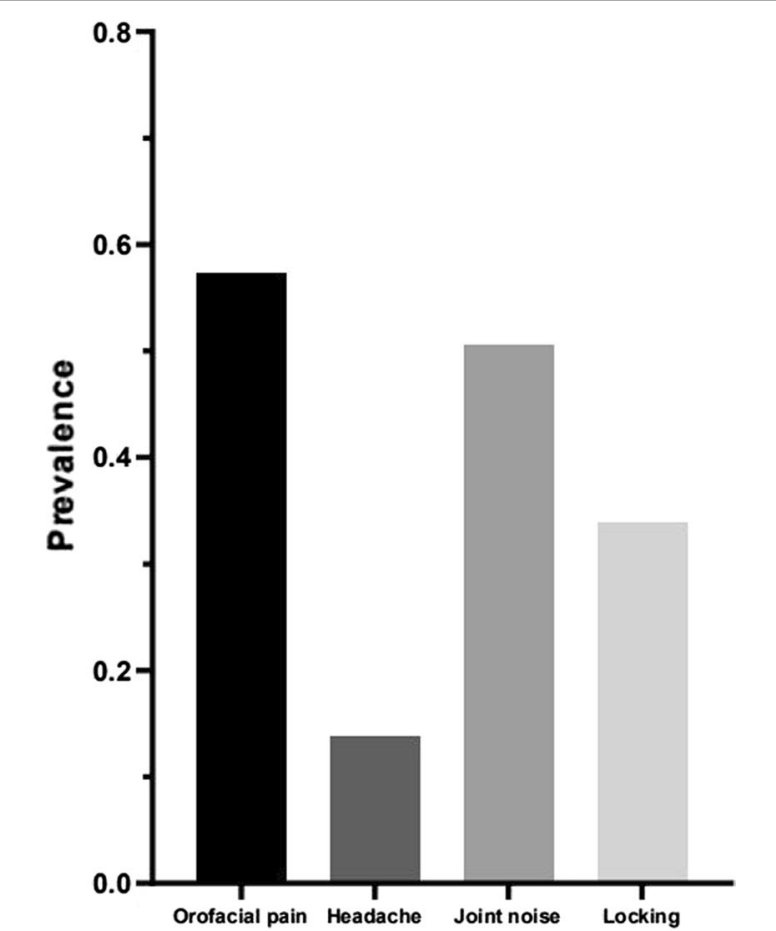

Fig. 1 Prevalence of temporomandibular disorder symptoms in the total participants
Table 1 Comparison of biological and psychological factors among participants without or with temporomandibular disorders (TMD)

\begin{tabular}{|c|c|c|c|}
\hline Characteristic & $\begin{array}{l}\text { Participants } \\
\text { without TMD } \\
(\mathrm{n}=515)\end{array}$ & $\begin{array}{l}\text { Participants } \\
\text { with TMD } \\
\text { (n=239) }\end{array}$ & $p$ value \\
\hline \multicolumn{4}{|l|}{ Biological factor } \\
\hline Age & $18(18,19)$ & $19(18,19)$ & 0.172 \\
\hline Female & $281(54.6)$ & $119(49.8)$ & 0.222 \\
\hline Sleep bruxism & $56(10.9)$ & $56(23.4)$ & $<0.001$ \\
\hline Awake bruxism & $16(3.1)$ & $18(7.5)$ & 0.006 \\
\hline Empty chewing & $39(7.6)$ & $50(20.9)$ & $<0.001$ \\
\hline Unilateral chewing & $172(33.4)$ & $135(56.5)$ & $<0.001$ \\
\hline Chewing gum & $135(26.2)$ & $81(33.9)$ & 0.030 \\
\hline \multicolumn{4}{|l|}{ Static occlusion } \\
\hline Angle's malocclusion & $185(35.9)$ & $92(38.4)$ & 0.464 \\
\hline Anterior teeth overbite & $213(41.3)$ & $139(58.1)$ & $<0.001$ \\
\hline Anterior teeth overjet & $163(31.7)$ & $102(42.7)$ & 0.003 \\
\hline $\begin{array}{l}\text { Posterior teeth } \\
\text { crossbite }\end{array}$ & $10(1.9)$ & $2(0.8)$ & 0.357 \\
\hline Posterior scissor bite & $18(3.5)$ & $14(5.9)$ & 0.173 \\
\hline \multicolumn{4}{|l|}{ Psychological factor } \\
\hline \multicolumn{4}{|l|}{ Depression } \\
\hline Prevalence & $232(45.0)$ & $167(69.9)$ & $<0.001$ \\
\hline Average score & $4(3,7)$ & $7(4,9)$ & $<0.001$ \\
\hline \multicolumn{4}{|l|}{ Anxiety } \\
\hline Prevalence & $163(31.7)$ & $166(69.5)$ & $<0.001$ \\
\hline Average score & $3(1,5)$ & $6(4,7)$ & $<0.001$ \\
\hline \multicolumn{4}{|l|}{ Sleep disturbance } \\
\hline Prevalence & $159(30.9)$ & $123(51.5)$ & $<0.001$ \\
\hline Average score & $5(3,6)$ & $6(4,7)$ & $<0.001$ \\
\hline
\end{tabular}

depression, anxiety, and sleep disturbance was observed in patients with TMD.

Furthermore, the association between TMD and biological and psychological characteristics was analyzed (Table 2). Students with TMD had a significant correlation with sleep bruxism, awake bruxism, empty chewing, unilateral chewing, chewing gum. And in occlusion aspects, anterior teeth overbite and anterior teeth overjet were correlated with TMD. Moreover, a significant correlation was also noted between TMD and depression, anxiety, and sleep disturbance. In addition, the influence of biological and psychological factors on different types of TMDs was verified. As shown in Table 3, there were 37, 160, and 42 people in the study with groups of the pain-related TMD, the intra-articular TMD, and the combined TMD, respectively. In the pain-related TMD group, it was only positively associated with sleep bruxism, unilateral chewing, anterior teeth overbite, depression, and anxiety. In the intra-articular TMD group, it was positive 
Table 2 Variables and risk factors for temporomandibular disorders (TMD)

\begin{tabular}{|c|c|c|c|c|c|c|c|c|}
\hline Biological factor & $r$ & $p$ value & Static occlusion & $r$ & $p$ value & Psychological factor & $r$ & $p$ value \\
\hline Age & 0.050 & 0.172 & Angle's malocclusion & -0.061 & 0.320 & Depression & 0.231 & $<0.001$ \\
\hline Gender & 0.044 & 0.222 & Anterior teeth overbite & 0.166 & $<0.001$ & Anxiety & 0.355 & $<0.001$ \\
\hline Sleep bruxism & 0.164 & $<0.001$ & Anterior teeth overjet & 0.110 & 0.002 & Sleep disturbance & 0.184 & $<0.001$ \\
\hline Awake bruxism & 0.099 & 0.006 & Posterior teeth crossbite & -0.041 & 0.260 & & & \\
\hline Empty chewing & 0.192 & $<0.001$ & Posterior scissor bite & 0.055 & 0.135 & & & \\
\hline Unilateral chewing & 0.219 & $<0.001$ & & & & & & \\
\hline Chewing gum & 0.079 & 0.030 & & & & & & \\
\hline
\end{tabular}

Table 3 Correlations of biological and psychological factors among different classifications of temporomandibular disorders (TMD)

\begin{tabular}{|c|c|c|c|c|c|c|}
\hline & \multicolumn{2}{|c|}{ Pain-related TMD $(n=37)$} & \multicolumn{2}{|c|}{ Intra-articular TMD $(n=160)$} & \multicolumn{2}{|c|}{ Combined TMD ( $n=42)$} \\
\hline & $r$ & $p$ value & $r$ & $p$ value & $r$ & $p$ value \\
\hline \multicolumn{7}{|l|}{ Biological factor } \\
\hline Age & 0.013 & 0.711 & 0.020 & 0.585 & 0.062 & 0.091 \\
\hline Gender & 0.008 & 0.832 & 0.097 & 0.008 & 0.089 & 0.014 \\
\hline Sleep bruxism & 0.078 & 0.033 & 0.075 & 0.039 & 0.126 & 0.001 \\
\hline Awake bruxism & 0.039 & 0.280 & 0.075 & 0.040 & 0.031 & 0.390 \\
\hline Empty chewing & 0.031 & 0.394 & 0.112 & 0.002 & 0.162 & $<0.001$ \\
\hline Unilateral chewing & 0.074 & 0.042 & 0.118 & 0.001 & 0.164 & $<0.001$ \\
\hline Chewing gum & 0.033 & 0.371 & 0.037 & 0.310 & 0.064 & 0.081 \\
\hline \multicolumn{7}{|l|}{ Static occlusion } \\
\hline Angle's malocclusion & 0.030 & 0.567 & 0.066 & 0.078 & 0.051 & 0.764 \\
\hline Anterior teeth overbite & 0.099 & 0.006 & 0.096 & 0.008 & 0.071 & 0.050 \\
\hline Anterior teeth overjet & 0.060 & 0.097 & 0.078 & 0.031 & 0.027 & 0.451 \\
\hline Posterior teeth crossbite & 0.020 & 0.580 & -0.066 & 0.070 & 0.015 & 0.674 \\
\hline Posterior scissor bite & 0.013 & 0.720 & 0.003 & 0.926 & 0.092 & 0.051 \\
\hline \multicolumn{7}{|l|}{ Psychological factor } \\
\hline Depression & 0.116 & 0.001 & 0.119 & 0.001 & 0.148 & $<0.001$ \\
\hline Anxiety & 0.134 & $<0.001$ & 0.230 & $<0.001$ & 0.148 & $<0.001$ \\
\hline Sleep disturbance & 0.026 & 0.477 & 0.119 & 0.001 & 0.114 & 0.002 \\
\hline
\end{tabular}

correlated with gender, sleep bruxism, awake bruxism, empty chewing, unilateral chewing, anterior teeth overbite, anterior teeth overjet, depression, anxiety, and sleep disturbance. There were also significant positive correlations between combined TMD and gender, sleep bruxism, empty chewing, unilateral chewing, depression, anxiety, and sleep disturbance.

In addition, depression, anxiety, and sleep disturbance were divided as mild to severe according to PHQ-9, GAD-7, and PSQI, respectively (Fig. 2). There are $52.3 \%$ students with mild depression, $15.5 \%$ students with moderate depression, and $2.1 \%$ students with moderate-severe depression among the TMD group, which revealed significantly higher severity of depression in patients with TMD. The anxiety and sleep disturbance parameters showed a similar trend.

\section{Assessment of possible risk factors among TMD}

In order to find out the factors as independent factors of TMD, logistic regression analyses were performed. The univariate logistic regression showed that sleep bruxism, awake bruxism, empty chewing, unilateral chewing, chewing gum, anterior teeth overbite, anterior teeth overjet, depression, anxiety, and sleep disturbance were risk factors for TMD. According to the multiple logistic regression model, sleep bruxism, empty chewing, unilateral chewing, anterior teeth 


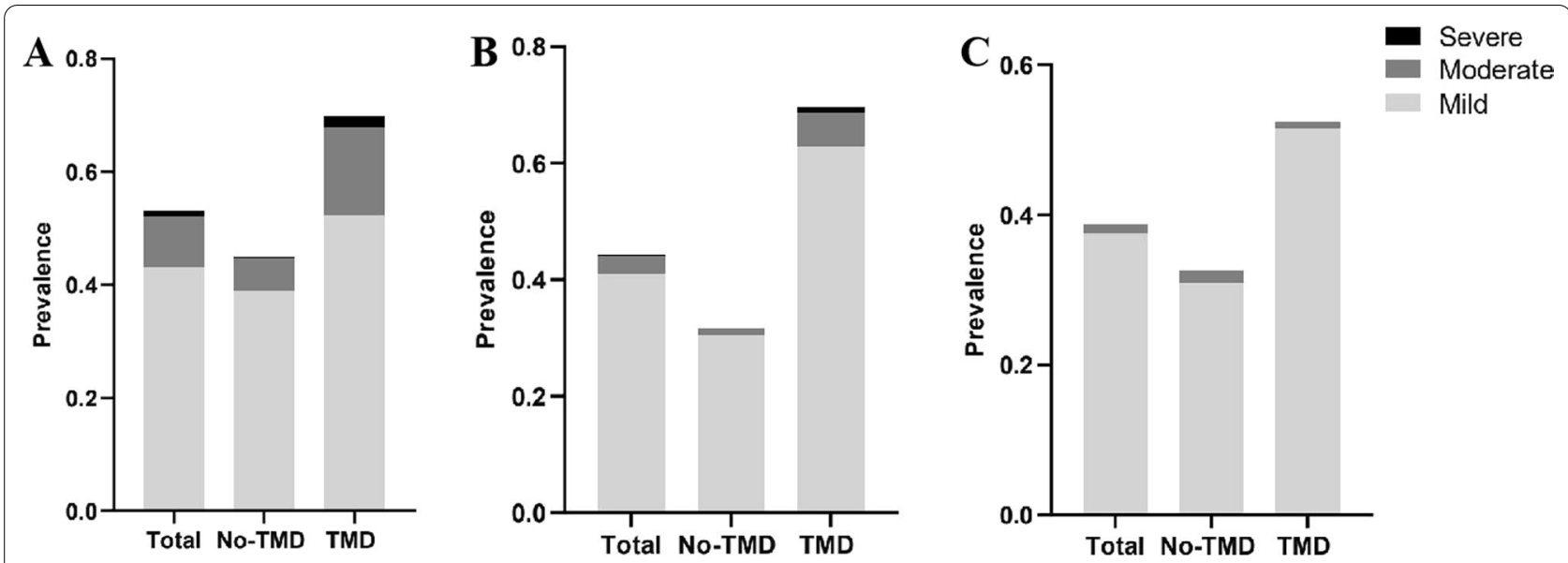

Fig. 2 Prevalence of psychological distress and sleep disturbance. Percentage of subjects with mild, moderate, and severe distress as shown, including depression (A), anxiety (B), and sleep disturbance (C)

Table 4 Univariate and multivariate logistic regression models of risk factors for temporomandibular disorders

\begin{tabular}{|c|c|c|c|c|}
\hline & \multicolumn{2}{|l|}{ Univariable } & \multicolumn{2}{|l|}{ Multivariable } \\
\hline & $\mathrm{HR}(95 \% \mathrm{Cl})$ & $p$ value & $\mathrm{HR}(95 \% \mathrm{Cl})$ & $p$ value \\
\hline \multicolumn{5}{|l|}{ Biological factor } \\
\hline Gender & $1.211(0.891,1.646)$ & 0.222 & & \\
\hline Sleep bruxism & $2.508(1.668,3.772)$ & $<0.001$ & $1.943(1.217,3.104)$ & 0.005 \\
\hline Awake bruxism & $2.540(1.272,5.073)$ & 0.008 & $1.284(0.567,2.908)$ & 0.548 \\
\hline Empty chewing & $3.229(2.056,5.071)$ & $<0.001$ & $2.489(1.484,4.173)$ & 0.001 \\
\hline Unilateral chewing & $2.589(1.890,3.545)$ & $<0.001$ & $1.679(1.178,2.393)$ & 0.004 \\
\hline Chewing gum & $1.443(1.035,2.001)$ & 0.030 & $0.977(0.668,1.429)$ & 0.906 \\
\hline \multicolumn{5}{|l|}{ Static occlusion } \\
\hline Angle's malocclusion & $1.276(0.785,3.461)$ & 0.364 & & \\
\hline Anterior teeth overbite & $1.234(1.115,1.367)$ & $<0.001$ & $1.193(1.014,1.403)$ & 0.033 \\
\hline Anterior teeth overjet & $1.262(1.073,1.485)$ & 0.005 & $1.017(0.786,1.317)$ & 0.895 \\
\hline Posterior teeth crossbite & $0.426(0.093,1.960)$ & 0.273 & & \\
\hline Posterior scissor bite & $1.718(0.840,3.515)$ & 0.138 & & \\
\hline \multicolumn{5}{|l|}{ Psychological factor } \\
\hline Depression & $2.829(2.042,3.921)$ & $<0.001$ & $1.656(1.143,2.400)$ & 0.008 \\
\hline Anxiety & $4.911(3.523,6.844)$ & $<0.001$ & $3.351(2.325,4.829)$ & $<0.001$ \\
\hline Sleep disturbance & $2.021(1.505,2.714)$ & $<0.001$ & $1.324(0.946,1.852)$ & 0.101 \\
\hline
\end{tabular}

$h$ hazard ratio, $\mathrm{Cl}$ confidence interval

overbite, depression, and anxiety were independent risk factors for TMD (Table 4).

\section{Discussion}

Academic stress is extremely high among medical university students in China due to educational standards, personal desires, and societal demands, which leads to psychological distress and sleep dysfunction and then contributes to TMD. Moreover, psychological distress also contributes to parafunctional habits like bruxism and abnormal chewing, which may associate with TMD. Thus, the current study aimed to investigate the effects of psychological aspects, parafunctional habits and malocclusions on TMD among medical university students in China.

In the present study, $31.7 \%$ of participants had at least one TMD symptom, which was considerably higher than the prevalence of TMD (only 10-20\%) in Asian youngers in the past century [22, 23]. It is worthy to further investigate the reasons resulting in a substantial increase 
in TMD prevalence among participants. One of the main findings in the present study was that psychological distress was more common in participants with TMD than those without TMD, of which depression, anxiety, and sleep disturbance in TMD patients were present in 69.9\%, $69.5 \%$, and $51.5 \%$, respectively. Moreover, depression and anxiety showed strong significant associations with TMD, which were independent risk factors for TMD. Similar results were reported by Anna and colleagues, a higher prevalence of depression, anxiety, and somatization among students with TMD symptoms in Poznań university of medical sciences [13]. Another research indicated that PHQ-9 and GAD-7 questionnaires for screening depression and anxiety should be considered in the diagnosis of TMD patients, which supported the present findings [15]. A possible explanation for associations between psychological diseases and TMD would be due to that psychological pressure leads to grinding and clenching, which exacerbates the masticatory muscle tension contributing to TMD [24-26]. Another major finding was that sleep disturbance was also more common in TMD patients, while it was only associated with intraarticular TMD. In another Chinese adolescent cohort research, a high prevalence of sleep disturbance and TMD symptoms were presented in senior high students, and sleep disturbance increased the risk of TMD symptoms [27]. Rener-Sitar and colleagues reported that TMD patients with pain possessed much poorer sleep quality, which indicated that sleep quality should be evaluated in TMD patients especially those with pain [28]. A circular relationship of mutual deleterious influences between TMD symptoms and sleep exists, thus sleep improvement should be targeted for negative effects on TMD.

Additionally, TMD symptoms were assessed concerning the presence of parafunctional habits including bruxism and abnormal chewing. The present study showed that subjects with TMD possessed a higher prevalence of certain parafunctional habits such as bruxism, empty chewing, unilateral chewing, and chewing gum, while sleep bruxism, empty chewing, and unilateral chewing were independent risks of developing TMD. A previous study also showed that oral parafunctional habits were commonly observed in college preparatory students, which were considered contributory factors for TMD symptoms [29]. Furthermore, TMD was more prevalent in cases with bruxism, while bruxism induced overloading of the temporomandibular joint due to degenerative changes [30]. In a survey of Turkish university students, unilateral chewing was significantly associated with TMD, which disturbed the rhythmic coordination of the jaw and neck muscles during chewing contributing to TMD symptoms [31]. Interestingly, those participating in the previous study with longer chewing cycles and length had more prevalence of experiencing TMD, which was consistent with the current findings [32]. It was explained that oral parafunctional habits might cause TMD symptoms due to the overloading of musculoskeletal structures. The relationship between TMD and occlusion is still controversial, and although some professionals considered that malocclusion is one of the factors for initiation of TMD. In the present study, TMD possessed a higher prevalence of anterior teeth overbite and anterior teeth overjet, which were independent risks of developing TMD. Thilander groups observed that TMDs were significantly associated with posterior crossbite, and suggested that malocclusions should be treated orthodontically in an early age to protect against TMD [33]. In contrast, Al-Ani and Špalj et al. found that symptoms of TMDs seemed to be poorly related to malocclusions [34, 35]. Although the evidence available does not allow to establish a unambiguous relationship between malocclusions and TMD, the effects of malocclusions in growing patients of TMDs deserve further investigation.

Apart from the noteworthy findings, there were some potential limitations in the present study. First, all participants were only from one medical university, the extended research incorporating students from other universities should be planned to increase sample size and truly reflect the situation of TMD in medical students. Second, the current study had not evaluated the imaging datas, which were mainly assessed through $\mathrm{X}$-ray or magnetic resonance imaging. It was difficult to comprehensively and accurately evaluate TMD conditions due to the lack of the imaging studies. Finally, the present study was a cross-sectional research, future studies should be considered a longitudinal study design to confirm these factors as the predictors to TMD (Additional file 1).

\section{Conclusions}

A high prevalence of TMD symptoms was reported among Chinese students at the university of medical sciences. TMD in those population was considered as multifactorial condition, in which psychological distress symptoms as depression, anxiety as well as sleep disturbance correlated with TMD. Some oral parafunctional habits and malocclusions showed association with TMD, mainly the presence of bruxism and abnormal chewing were potential risk factors to TMD.

\section{Supplementary Information}

The online version contains supplementary material available at https://doi. org/10.1186/s12903-021-01916-2.

Additional file 1. TMD Symptom Questionnaire. 


\section{Acknowledgements \\ Not applicable.}

\section{Authors' contributions}

JW: Conceptualization, Methodology, Writing an original draft, Visualization, Project administration. ZLH: Methodology, Writing review and editing. YC, YFC, and ZQP: Methodology, Editing, Supervision. YG: Conceptualization, Writing review and editing. All authors read and approved the final manuscript.

\section{Funding}

This study received no specific Grant from any funding agencies.

\section{Availability of data and material}

The datasets used and/or analysed during the current study are available from the corresponding author on reasonable request.

\section{Declarations}

\section{Ethics approval and consent to participate}

This study was approved by the ethical committee of the Zunyi Medical University (2019-H007). Written informed consent was obtained from all subjects.

\section{Consent for publication}

All authors consent for publication.

\section{Competing interests}

The authors declare no conflicts of interest.

\begin{abstract}
Author details
'Department of Stomatology, Huzhou Traditional Chinese Medicine Hospital, Zhejiang Chinese Medicinal University, Huzhou, Zhejiang, China. ${ }^{2}$ Guangzhou Key Laboratory of Basic and Applied Research of Oral Regenerative Medicine, Affiliated Stomatology Hospital of Guangzhou Medical University, Guangzhou, Guangdong, China. ${ }^{3}$ Department of Stomatology, Stomatological Hospital of Honghuagang, Zunyi, Guizhou, China. ${ }^{4}$ Department of Acupuncture, Huzhou Traditional Chinese Medicine Hospital, Zhejiang Chinese Medicinal University, Huzhou, Zhejiang, China. ${ }^{5}$ Department of Stomatology, Zhuhai Campus of Zunyi Medical University, 368 Jinwan Road, Zhuhai 519041, Guangdong Province, China.
\end{abstract}

Received: 16 August 2021 Accepted: 12 October 2021

Published online: 26 October 2021

\section{References}

1. Fernández-de-Las-Peñas C, Von Piekartz H. Clinical reasoning for the examination and physical therapy treatment of temporomandibular disorders (TMD): a narrative literature review. J Clin Med. 2020;9(11):3686.

2. Slade GD, Ohrbach R, Greenspan JD, Fillingim RB, Bair E, Sanders AE, Dubner R, Diatchenko L, Meloto CB, Smith S, et al. Painful temporomandibular disorder: decade of discovery from OPPERA studies. J Dent Res. 2016;95(10):1084-92.

3. List T, Jensen RH. Temporomandibular disorders: old ideas and new concepts. Cephalalgia. 2017;37(7):692-704

4. Ohrbach R, Dworkin SF. The evolution of TMD diagnosis: past, present future. J Dent Res. 2016:95(10):1093-101.

5. Visscher CM, Baad-Hansen L, Durham J, Goulet J-P, Michelotti A, Roldán Barraza C, Häggman-Henrikson B, Ekberg E, Raphael KG. Benefits of implementing pain-related disability and psychological assessment in dental practice for patients with temporomandibular pain and other oral health conditions. J Am Dent Assoc. 2018;149(6):422-31.

6. Fillingim RB, Ohrbach R, Greenspan JD, Knott C, Diatchenko L, Dubner R, Bair E, Baraian C, Mack N, Slade GD, et al. Psychological factors associated with development of TMD: the OPPERA prospective cohort study. J Pain. 2013;14(12 Suppl):T75-90.

7. Ohrbach R, Slade GD, Bair E, Rathnayaka N, Diatchenko L, Greenspan JD, Maixner W, Fillingim RB. Premorbid and concurrent predictors of TMD onset and persistence. Eur J Pain. 2020;24(1):145-58.
8. Jiménez-Silva A, Peña-Durán C, Tobar-Reyes J, Frugone-Zambra R. Sleep and awake bruxism in adults and its relationship with temporomandibular disorders: a systematic review from 2003 to 2014. Acta Odontol Scand. 2017;75(1):36-58

9. Ohlmann B, Waldecker M, Leckel M, Bömicke W, Behnisch R, Rammelsberg P, Schmitter M. Correlations between sleep bruxism and temporomandibular disorders. J Clin Med. 2020;9(2):611.

10. Shu J, Zhang Y, Chong DYR, Liu Z. Effects on loads in temporomandibular joints for patients with mandibular asymmetry before and after orthognathic surgeries under the unilateral molar clenching. Biomech Model Mechanobiol. 2020;19(2):533-41.

11. Khayat N, Winocur E, Emodi Perelman A, Friedman-Rubin P, Gafni Y, Shpack N. The prevalence of posterior crossbite, deep bite, and sleep or awake bruxism in temporomandibular disorder (TMD) patients compared to a non-TMD population: a retrospective study. Cranio. 2021:39(5):398-404.

12. Giray B, Sadry S. Modifications in Class I and Class II Div. 1 malocclusion during orthodontic treatment and their association with TMD problems. Cranio. 2021;39(1):65-73.

13. Sójka A, Stelcer B, Roy M, Mojs E, Pryliński M. Is there a relationship between psychological factors and TMD? Brain Behav. 2019;9(9):e01360.

14. Canales GDLT, Guarda-Nardini L, Rizzatti-Barbosa CM, Conti PCR, Manfredini D. Distribution of depression, somatization and pain-related impairment in patients with chronic temporomandibular disorders. J Appl Oral Sci. 2019;27:e20180210.

15. Simoen L, Van den Berghe L, Jacquet W, Marks L. Depression and anxiety levels in patients with temporomandibular disorders: comparison with the general population. Clin Oral Investig. 2020;24(11):3939-45.

16. Dreweck FDS, Soares S, Duarte J, Conti PCR, De Luca Canto G, Luís Porporatti $A$. Association between painful temporomandibular disorders and sleep quality: a systematic review. J Oral Rehabil. 2020;47(8):1041-51.

17. Natu VP, Yap AUJ, Su MH, Irfan Ali NM, Ansari A. Temporomandibular disorder symptoms and their association with quality of life, emotional states and sleep quality in South-East Asian youths. J Oral Rehabil. 2018;45(10):756-63.

18. Schiffman E, Ohrbach R, Truelove E, Look J, Anderson G, Goulet J-P, List T, Svensson P, Gonzalez Y, Lobbezoo F, et al. Diagnostic criteria for temporomandibular disorders (DC/TMD) for clinical and research applications: recommendations of the International RDC/TMD Consortium Network* and Orofacial Pain Special Interest Group ${ }^{\dagger}$. J Oral Facial Pain Headache. 2014;28(1):6.

19. Levis B, Benedetti A, Thombs BD. Accuracy of patient health questionnaire-9 (PHQ-9) for screening to detect major depression: individual participant data meta-analysis. BMJ. 2019;365:11476.

20. Toussaint A, Hüsing P, Gumz A, Wingenfeld K, Härter M, Schramm E, Löwe B. Sensitivity to change and minimal clinically important difference of the 7-item generalized anxiety disorder questionnaire (GAD-7). J Affect Disord. 2020;265:395-401.

21. Buysse DJ, Reynolds CF, Monk TH, Berman SR, Kupfer DJ. The Pittsburgh Sleep Quality Index: a new instrument for psychiatric practice and research. Psychiatry Res. 1989;28(2):193-213.

22. Verdonck A, Takada K, Kitai N, Kuriama R, Yasuda Y, Carels C, Sakuda M. The prevalence of cardinal TMJ dysfunction symptoms and its relationship to occlusal factors in Japanese female adolescents. J Oral Rehabil. 1994;21(6):687-97.

23. Deng YM, Fu MK, Hägg U. Prevalence of temporomandibular joint dysfunction (TMJD) in Chinese children and adolescents. A cross-sectional epidemiological study. Eur J Orthod. 1995;17(4):305-9.

24. Al-Khotani A, Naimi-Akbar A, Gjelset M, Albadawi E, Bello L, HedenbergMagnusson B, Christidis N. The associations between psychosocial aspects and TMD-pain related aspects in children and adolescents. J Headache Pain. 2016:17:30.

25. Karibe H, Shimazu K, Okamoto A, Kawakami T, Kato Y, Warita-Naoi S. Prevalence and association of self-reported anxiety, pain, and oral parafunctional habits with temporomandibular disorders in Japanese children and adolescents: a cross-sectional survey. BMC Oral Health. 2015;15:8.

26. de Paiva Bertoli FM, Bruzamolin CD, de Almeida Kranz GO, Losso EM, Brancher JA, de Souza JF. Anxiety and malocclusion are associated with temporomandibular disorders in adolescents diagnosed by RDC/TMD. A cross-sectional study. J Oral Rehabil. 2018, 45(10):747-55. 
27. Lei J, Fu J, Yap AUJ, Fu K-Y. Temporomandibular disorders symptoms in Asian adolescents and their association with sleep quality and psychological distress. Cranio. 2016;34(4):242-9.

28. Rener-Sitar K, John MT, Pusalavidyasagar SS, Bandyopadhyay D, Schiffman EL. Sleep quality in temporomandibular disorder cases. Sleep Med. 2016;25:105-12.

29. Paulino MR, Moreira VG, Lemos GA, Silva PLP, Bonan PRF, Batista AUD. Prevalence of signs and symptoms of temporomandibular disorders in college preparatory students: associations with emotional factors, parafunctional habits, and impact on quality of life. Cien Saude Colet. 2018:23(1):173-86.

30. Naeije M, Te Veldhuis AH, Te Veldhuis EC, Visscher CM, Lobbezoo F. Disc displacement within the human temporomandibular joint: a systematic review of a'noisy annoyance'. J Oral Rehabil. 2013;40(2):139-58.

31. Yalçın Yeler D, Yılmaz N, Koraltan M, Aydın E. A survey on the potential relationships between TMD, possible sleep bruxism, unilateral chewing, and occlusal factors in Turkish university students. Cranio. 2017;35(5):308-14.
32. Fassicollo CE, Garcia DM, Machado BCZ, de Felício CM. Changes in jaw and neck muscle coactivation and coordination in patients with chronic painful TMD disk displacement with reduction during chewing. Physiol Behav. 2021;230:113267.

33. Thilander B, Rubio G, Pena L, de Mayorga C. Prevalence of temporomandibular dysfunction and its association with malocclusion in children and adolescents: an epidemiologic study related to specified stages of dental development. Angle Orthod. 2002;72(2):146-54.

34. Al-Ani Z. Occlusion and temporomandibular disorders: a long-standing controversy in dentistry. Prim Dent J. 2020;9(1):43-8.

35. Špalj S, Šlaj M, Athanasiou AE, Žak I, Šimunović M, Šlaj M. Temporomandibular disorders and orthodontic treatment need in orthodontically untreated children and adolescents. Coll Antropol. 2015;39(1):151-8.

\section{Publisher's Note}

Springer Nature remains neutral with regard to jurisdictional claims in published maps and institutional affiliations.
Ready to submit your research? Choose BMC and benefit from:

- fast, convenient online submission

- thorough peer review by experienced researchers in your field

- rapid publication on acceptance

- support for research data, including large and complex data types

- gold Open Access which fosters wider collaboration and increased citations

- maximum visibility for your research: over $100 \mathrm{M}$ website views per year

At BMC, research is always in progress.

Learn more biomedcentral.com/submissions 